\title{
Assembleia de aves na área urbana do município de Pelotas, Rio Grande do Sul, Brasil
}

\author{
Anne Gomes Sacco ${ }^{I}$, Fabiane Borba Bergmann ${ }^{2}$ \& Ana Maria Rui ${ }^{3,4}$ \\ ${ }^{1}$ Laboratório de Ecologia de Populações e Comunidades, Centro de Ecologia, Instituto de Biociências, \\ Universidade Federal do Rio Grande do Sul-UFRGS, Av. Bento Gonçalves, 9500, CP 15007, \\ CEP 91501-970, Porto Alegre, RS, Brasil \\ ${ }^{2}$ Centro de Ciências Naturais e Exatas, Universidade Federal de Santa Maria-UFSM, Av. Roraima, s/n, \\ Camobi, CEP 97105-900, Santa Maria, RS, Brasil \\ ${ }^{3}$ Departamento de Ecologia, Zoologia e Genética, Instituto de Biologia, Universidade Federal de Pelotas- \\ UFPel, CP 354, Campus Universitário Capão do Leão, CEP 96001-970, Pelotas, RS, Brasil \\ ${ }^{4}$ Autor para correspondência: Ana MariaRui,e-mail: ana.rui@ufpel.edu.br
}

SACCO, A.G., BERGMANN, F.B. \& RUI, A.M. Bird assemblages in the urban area in the city of Pelotas, Rio Grande do Sul, Brazil. Biota Neotrop. 13(2): http://www.biotaneotropica.org.br/v13n2/en/abstract?inven tory+bn01113022013

\begin{abstract}
Urbanization causes changes in the composition and diversity of biotic communities. The goals of this work are to present a list of bird species that use the urban area in the city of Pelotas (RS), in the southernmost end of Brazil, and to describe the bird assemblages structure underlining the richness, composition, and relative abundance of species and their seasonal variability. Data collection took place between October 2007 and September 2008 , at 216 fixed points, distributed in groups of 6 equidistant points at each 200 meters in streets with varied levels of urbanization, sampled once a season. The observer stayed for 8 minutes at each point, resulting in a sampling effort of 28.8 hours each season and 115.2 hours during the year. A total of 9,595 contacts of 84 bird species were found, belonging to 34 families and 14 orders. In regard to relative abundance, 72 species presented less than 200 contacts; 7 presented between 200 and 1,000; and 4 presented over 1,000 contacts. The species with more than 1,000 contacts were Furnarius rufus, Columba livia, Myiopsitta monachus, and Pitangus sulphuratus, accounting for 5,136 contacts. In all 84 species, 67 were present in less than $20 \%$ of the sampled points; 7 were registered at between $20-40 \%$ and between $40-60 \%$ of the points; and 3 were sighted at between $80-100 \%$ of the observation points. Species with the highest frequency of occurrence were P. sulphuratus, Passer domesticus, and F. rufus. Total richness ranged from 55 species in the fall to 61 in the winter. Of the 84 species, 21 were present in 1 season, 17 in 2, 9 in 3, and 37 were present in all four seasons. The season with the highest relative abundance was spring, with 2,936 contacts; the one with the least abundance was autumn, with 2,149 contacts. The bird assemblages presented a representative richness relative to the region, though very few species are abundant and well distributed. Such pattern may be a reflection of the low quality and heterogeneity of the urban matrix, which has few parks and green areas, no reserves with native vegetation and lakes, and a low degree of tree coverage. Keywords: relative abundance, Pampa Biome, richness, seasonality, urbanization.
\end{abstract}

SACCO, A.G., BERGMANN, F.B. \& RUI, A.M. Assembleia de aves na área urbana do município de Pelotas, Rio Grande do Sul, Brasil. Biota Neotrop. 13(2): http://www.biotaneotropica.org.br/v13n2/pt/abstract?invent ory+bn01113022013

Resumo: A urbanização altera a composição e diversidade das comunidades bióticas. Os objetivos do trabalho são apresentar a lista de espécies de aves que utilizam a área urbana do município de Pelotas (RS), no extremo sul do Brasil, e descrever a estrutura da assembleia com ênfase na riqueza, composição e abundância relativa de espécies e suas variações sazonais. A coleta de dados ocorreu entre outubro de 2007 e setembro de 2008, em 216 pontos fixos de observação distribuídos em grupos de seis pontos em ruas com todos os graus de urbanização, que foram amostrados uma vez por estação. O observador permaneceu oito minutos em cada um dos pontos, o que resultou no esforço amostral de 28,8 horas em cada estação e 115,2 horas no ano. Foram registrados 9.595 contatos de 84 espécies de aves pertencentes a 34 famílias e 14 ordens. Quanto à abundância relativa, 72 espécies apresentaram menos de 200 contatos; sete apresentaram entre 200 e 1000; e quatro apresentaram mais de 1000 contatos. As espécies com mais de 1000 contatos foram Furnarius rufus, Columba livia, Myiopsitta monachus e Pitangus sulphuratus, totalizando 5136 contatos. Das 84 espécies, 67 estiveram presentes em menos do que $20 \%$ dos pontos amostrados; sete foram registradas entre 20 e $40 \%$ e entre 40 e $60 \%$ dos pontos; e três foram visualizadas entre 80 e $100 \%$ dos pontos. As espécies com maior frequência de ocorrência foram P. sulphuratus, Passer domesticus e F. rufus. A riqueza variou de 55 espécies, no outono, até 61, no inverno. Das 84 espécies, 21 estiveram presentes em uma estação, 17 em duas, nove em três e 37 estiveram presentes em todas as estações. A estação em que se obteve maior abundância relativa foi a primavera, com 2936 contatos, e a menor foi o outono, com 2149 contatos. A assembleia de aves apresentou riqueza representativa se comparada com a região, porém, poucas espécies são abundantes e bem distribuídas. Esse padrão pode ser reflexo da baixa qualidade e heterogeneidade da matriz urbana, que possui poucas praças e parques, não possui áreas de reserva de vegetação nativa e lagos e possui baixo grau de arborização.

Palavras-chave: abundância relativa, Bioma Pampa, riqueza, sazonalidade, urbanização. 


\section{Introdução}

As cidades são habitats homogêneos e a urbanização é a atividade humana que promove a mais intensa homogeneização biótica (Blair 2001, McKinney 2006). A urbanização afeta a riqueza de espécies, que pode aumentar ou diminuir dependendo de variáveis como o grupo taxonômico, a escala espacial da análise e a intensidade da urbanização (McKinney 2008). McKinney (2008) revisou 105 estudos sobre o efeito da urbanização em espécies de mamíferos, répteis, anfíbios, invertebrados e plantas. Para todos os grupos, a riqueza de espécies tende a ser reduzida em áreas de extrema urbanização, porém, os efeitos de níveis moderados de urbanização variam significativamente entre grupos. Para as plantas, $65 \%$ dos trabalhos revisados tinham resultados que indicavam aumento de riqueza de espécies em áreas com urbanização moderada e apenas $12 \%$ dos trabalhos de vertebrados não voadores obtiveram os mesmos resultados.

O número de estudos que descrevem as respostas das aves à urbanização é grande e está aumentando (para uma revisão ver Chace \& Walsh 2006), o que está relacionado a sua grande riqueza, abundância, diversidade morfológica e ecológica e ao fato de serem facilmente amostradas e monitoradas, já que em sua maioria podem ser identificadas por um observador bem treinado. Além disso, as aves são muito sensíveis às mudanças na estrutura e composição do habitat e, portanto, excelentes indicadores de mudanças e tensões no ecossistema urbano (Savard \& Falls 1982, Clergeau et al. 1998).

Atualmente, quase a totalidade do conjunto de dados disponíveis sobre diversidade de aves em áreas urbanas e efeitos da urbanização é proveniente de trabalhos realizados em países da Europa e da América do Norte. Desta forma, as generalizações sobre o assunto foram baseadas na fauna de aves destas regiões do hemisfério norte. Chace \& Walsh (2006) revisaram os efeitos da urbanização sobre populações e comunidades de aves nativas e compilaram os principais padrões. Segundo estes autores, as comunidades de aves urbanas em habitats distintos mostram diferenças mais profundas em áreas menos alteradas do que em áreas alteradas; a urbanização tende a favorecer espécies onívoras, granívoras e espécies que nidificam em cavidades; e a intensificação da urbanização aumenta a biomassa de aves e causa uma diminuição da riqueza. Clergeau et al. (2006) avaliou 19 cidades na Europa e constatou que o número total de espécies de aves decresce significativamente de áreas suburbanas e periurbanas para setores centrais destas cidades.

Foram registradas 1.832 espécies de aves no Brasil (Cômite Brasileiro de Registros Ornitológicos 2011). No Rio Grande do Sul (RS), ocorrem 661 espécies, sendo que este número deve crescer ao longo da próxima década (Bencke et al. 2010). A riqueza no Estado, segundo Belton (2004), resulta da heterogeneidade de habitats e de sua localização geográfica privilegiada, dentro da zona de transição entre as florestas brasileiras e as regiões de campos sulinos do continente americano.

No Brasil, a grande maioria dos estudos realizados em cidades contemplando aves foi executada em áreas verdes no interior da matriz urbana, em habitats como fragmentos florestais, parques, praças ou entorno de lagos. Entre estes estudos estão os de MatarazzoNeuberger (1992, 1995), Monteiro \& Brandão (1995), Alves \& Pereira (1998), Borges \& Guilherme (2000), Krügel \& Anjos (2000), Franchin \& Marçal-Júnior (2004), Silva \& Blamires (2007) e Fuscaldi \& Loures-Ribeiro (2008). No RS, também foram realizados alguns trabalhos nesse tipo de habitat, tal como os de Mendonça-Lima \& Fontana (2000), Scherer et al. (2005), Silva (2006, 2007) e Paetzold \& Querol (2008). Alguns estudos trataram mais especificamente da resposta da assembleia ou de algumas espécies de aves a urbanização: Ruszczyk et al. (1987) analisaram os padrões de distribuição de oito espécies no gradiente de urbanização em Porto Alegre, no Rio Grande do Sul; Amâncio et al. (2008) estudaram o potencial de Columba livia e Pitangus sulphuratus como indicadoras de qualidade ambiental na cidade de Uberlândia, em Minas Gerais; Fontana et al. (2011) avaliaram os padrões de riqueza e de densidade de espécies e as variáveis que afetam a assembleia de aves em Porto Alegre (RS); e Reis et al. (2012) abordaram a questão de como o processo de urbanização afeta a riqueza de espécies de aves em Palmas (TO), uma cidade no Cerrado do Brasil.

Antes da implementação de políticas de gestão para reduzir os impactos urbanos sobre as comunidades de aves é necessário a existência de estudo e conhecimento local, incluindo monitoramentos de longo prazo (Chace \& Walsh 2006). Os dados disponibilizados neste estudo constituem a base para análises relacionando a estrutura da assembleia de aves com diferentes níveis de urbanização (espaço) e com alterações da qualidade da matriz urbana (tempo). Além disso, foram detectadas as espécies frequentes e abundantes na matriz urbana, que podem ser utilizadas para monitoramentos populacionais de longo prazo. No conjunto, os dados disponibilizados podem ser utilizados em projetos de planejamento urbano para manter espécies, controlar espécies e manter a diversidade de espécies de aves na matriz urbana.

Este trabalho tem como objetivos determinar a riqueza, composição e abundância relativa das espécies de aves na área urbana do município de Pelotas (RS), sul do Brasil, e avaliar as variações sazonais da assembleia.

\section{Material e Métodos}

\section{1. Área de estudo}

O estudo foi realizado na área urbana do município de Pelotas (3146'19" S e 52²0'33” W), situado no RS, no extremo sul do Brasil (Figura 1). O município está localizado na região geomorfológica da Planície Costeira, à altitude de sete metros do nível do mar, às margens do Canal São Gonçalo, um canal navegável que liga as Lagoas dos Patos e Mirim, as maiores lagoas do Brasil. A população estimada é de 339.934 mil habitantes e a área que ocupa é de $1609 \mathrm{~km}^{2}$ (Instituto... 2007). O município está inserido no Bioma Pampa, na fisionomia das Formações Pioneiras (vegetação com influência fluvial e/ou lacustre) recebendo influência, devido a sua proximidade, da fisionomia da Floresta Estacional Semidecidual, situada a oeste (Instituto... 1986). O clima de Pelotas, segundo o modelo de classificação climática de Köppen, é do tipo Cfa (Clima Temperado Úmido com Verão Quente). A média anual da temperatura é de $17,8^{\circ} \mathrm{C}$ e a média da precipitação pluviométrica anual é de $1366,9 \mathrm{~mm}$ (Embrapa 2008). No período do estudo, a pluviosidade e as temperaturas médias estacionais foram respectivamente: $329,2 \mathrm{~mm}$ e $19,96{ }^{\circ} \mathrm{C}$ na primavera; $323,4 \mathrm{~mm}$ e $22,63{ }^{\circ} \mathrm{C}$ no verão; $321,9 \mathrm{~mm}$ e $15,03{ }^{\circ} \mathrm{C}$ no outono; e $392,2 \mathrm{~mm}$ e $14,06{ }^{\circ} \mathrm{C}$ no inverno (Embrapa 2008).

\section{Metodologia}

Para a avaliação da avifauna foi utilizado o método de contagem por pontos (baseado em Bibby et al. 1992). Foram marcados 216 pontos fixos de observação de aves distribuídos em grupos de seis pontos equidistantes cerca de 200 metros em ruas da malha urbana do município. A distribuição dos pontos foi feita em toda a malha urbana, havendo o cuidado para que todos os graus de urbanização e todos os tipos de habitats fossem amostrados, incluindo núcleo urbano, periferia do núcleo urbano e a área periurbana. A coleta de dados ocorreu durante doze meses, no período de outubro de 2007 a setembro de 2008. Foram amostrados 12 pontos por dia e 72 pontos por mês em seis diferentes dias de coleta de dados. Desta forma, todos 


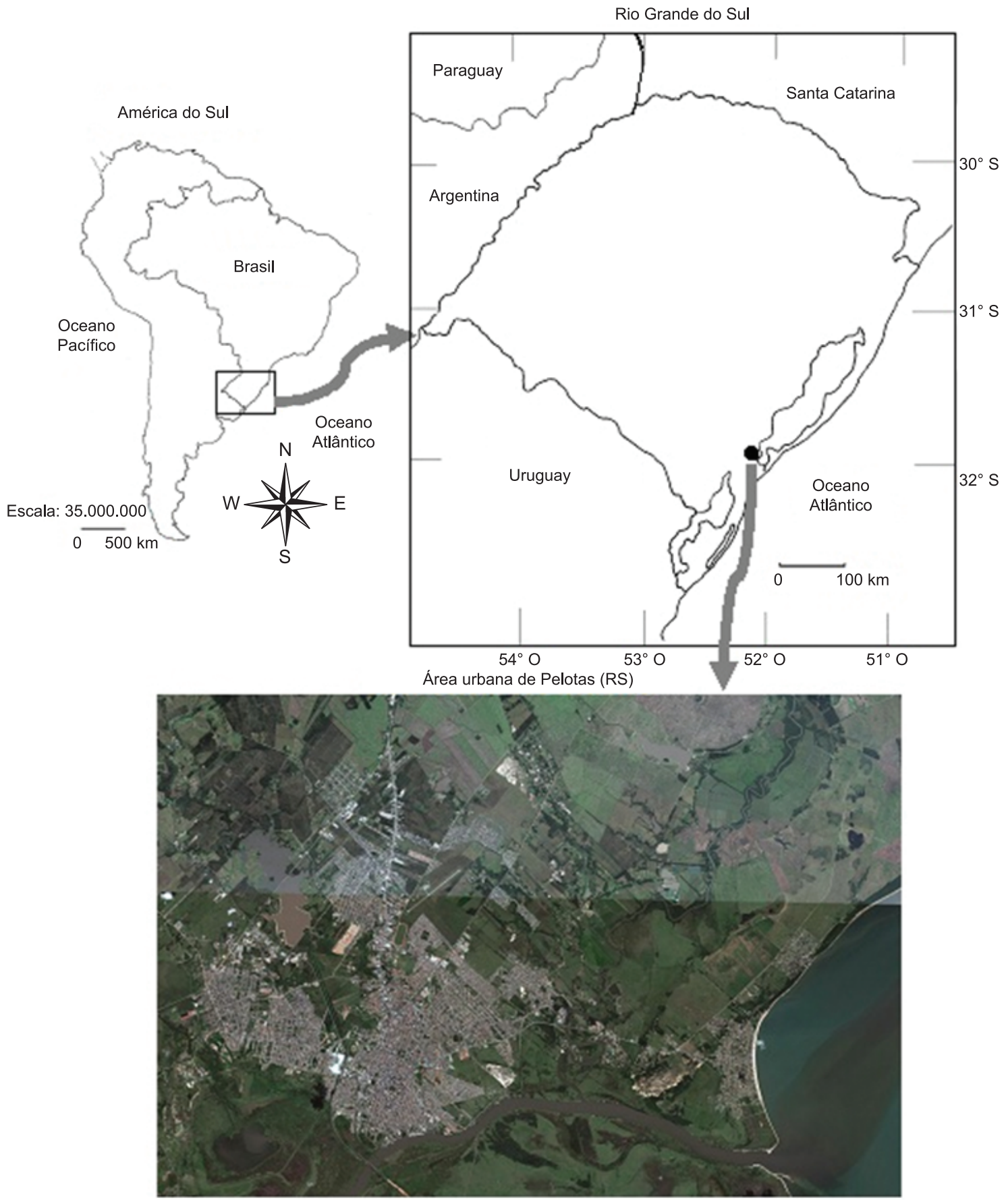

Figura 1. Localização do município de Pelotas, Rio Grande do Sul, Brasil, e disposição da área urbana à margem da Lagoa dos Patos.

Figure 1. Location of the municipality of Pelotas, Rio Grande do Sul, Brazil, and disposition of the urban area outside the Lagoa dos Patos.

os 216 pontos foram amostrados a cada três meses, permitindo uma análise estacional. Todas as observações foram realizadas no turno da manhã, iniciando por volta de 30 minutos após o amanhecer. As amostragens foram realizadas sempre pelo mesmo observador (A.G. Sacco), que permanecia oito minutos em cada um dos pontos, o que resultou no esforço amostral de 28,8 horas em cada estação e 115,2 horas no ano. Os registros das aves foram visuais com auxílio de binóculo, sendo contabilizado o número de contatos com cada espécie em um raio de 50 metros em torno do observador. Foram consideradas as vocalizações somente nos casos em que o contato visual com o indivíduo foi impossível e, nesse caso, foi contabilizado apenas um contato por espécie em cada ponto.

A assembleia de aves foi avaliada quanto à riqueza de espécies nas estações e no ano e foram calculadas a abundância relativa (AR) e a frequência de ocorrência (FO) anuais de cada espécie na amostra. A AR é a porcentagem de registros de ocorrência de uma espécie na amostra em relação ao número total de registros de ocorrência obtidos e a FO é a porcentagem do número de pontos em que cada espécie foi registrada em relação ao número total de pontos amostrados. Para verificar se o número de unidades amostrais foi suficiente para obter 
uma amostragem significativa da riqueza na área, foi construída a curva de acumulação de espécies (rarefação baseada nas unidades amostrais) no software PAST (versão 2.09) (Hammer et al. 2001).

Os indivíduos de Passer domesticus (Linnaeus, 1758) não foram contabilizados, já que esta é uma espécie extremamente abundante em ambiente urbano, o que torna difícil sua contagem exata. Porém, a espécie foi considerada para a análise de riqueza.

\section{Resultados}

Foram registrados 9.595 contatos de 84 espécies de aves pertencentes a 34 famílias e 14 ordens. A ordem dos Passeriformes foi dominante em relação ao número de espécies $(\mathrm{n}=39 ; 46,4 \%)$, sendo que as famílias mais representativas foram Tyrannidae $(n=8 ; 9,5 \%)$, Emberezidae $(n=6 ; 7,1 \%)$ e Icteridae $(n=6 ; 7,1 \%)$. Já em relação às ordens Não-Passeriformes destacaram-se os Ciconiformes $(\mathrm{n}=9$; $10,7 \%)$, Charadriiformes ( $n=7 ; 8,3 \%)$ e Columbiformes $(n=6 ; 7,1 \%)$ (Tabela 1). A curva de acumulação de espécies não atingiu a assíntota, o que indica que a riqueza é maior do que a que foi obtida. Porém, o esforço foi eficiente em obter uma amostragem significativa da riqueza de aves, pois com a metade do número de pontos já havia sido amostrado em torno de $85 \%$ do número total de espécies encontradas na área de estudo (Figura 2).

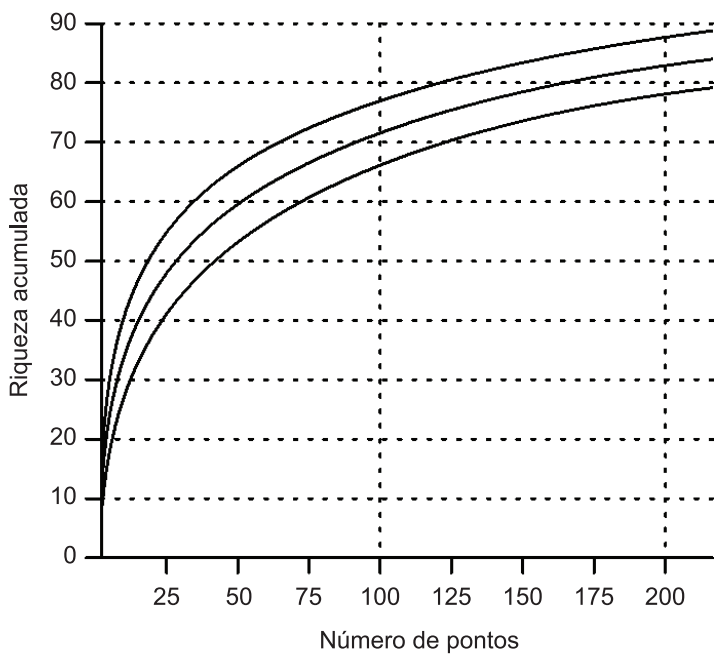

Figura 2. Curva de acumulação de espécies nos 216 pontos amostrais obtida de outubro de 2007 até setembro de 2008, em Pelotas (RS), Brasil.

Figure 2. Accumulation curve of species in 216 sampling points obtained from October 2007 until September 2008, in Pelotas (RS), Brazil.

Tabela 1. Lista das espécies de aves registradas de outubro de 2007 até setembro de 2008, na área urbana de Pelotas (RS), Brasil. Para cada espécie é informado o número de contatos nas estações e no ano, a abundância relativa (AR) e a frequência de ocorrência (FO) anuais. Nomenclatura e ordem taxonômica de acordo com Comitê Brasileiro de Registros Ornitológico (2011).

Table 1. Taxonomy list of bird species recorded from October 2007 until September 2008, in the urban area of Pelotas (RS), Brazil. For each species is reported the number of contacts in the seasons and year, the relative abundance (AR) and frequency of occurrence (FO) annually. Nomenclature and taxonomic order according to the Comitê Brasileiro de Registros Ornitológico (2011).

\begin{tabular}{|c|c|c|c|c|c|c|c|}
\hline \multirow{2}{*}{ Espécies } & \multicolumn{5}{|c|}{ Estações } & \multirow{2}{*}{$\operatorname{AR}(\%)$} & \multirow{2}{*}{ FO $(\%)$} \\
\hline & Primavera & Verão & Outono & Inverno & Total Anual & & \\
\hline \multicolumn{8}{|l|}{ Anseriformes } \\
\hline \multicolumn{8}{|l|}{ Anhimidae } \\
\hline Chauna torquata (Oken, 1816) & 0 & 3 & 2 & 0 & 5 & 0,05 & 0,9 \\
\hline \multicolumn{8}{|l|}{ Anatidae } \\
\hline Dendrocygna viduata (Linnaeus, 1766) & 21 & 0 & 0 & 2 & 23 & 0,24 & 1,4 \\
\hline Anas flavirostris (Vieillot, 1816) & 0 & 0 & 0 & 3 & 3 & 0,03 & 0,9 \\
\hline Anas versicolor (Vieillot, 1816) & 3 & 10 & 0 & 0 & 13 & 0,13 & 1,8 \\
\hline \multicolumn{8}{|l|}{ Pelecaniformes } \\
\hline \multicolumn{8}{|l|}{ Phalacrocoracidae } \\
\hline Phalacrocorax brasilianus (Gmelin, 1789) & 0 & 0 & 0 & 3 & 3 & 0,03 & 0,5 \\
\hline \multicolumn{8}{|l|}{ Ciconiiformes } \\
\hline \multicolumn{8}{|l|}{ Ardeidae } \\
\hline Bubulcus ibis (Linnaeus, 1758) & 0 & 18 & 0 & 0 & 18 & 0,19 & 1,4 \\
\hline Ardea cocoi (Linnaeus, 1766) & 0 & 1 & 0 & 0 & 1 & 0,01 & 0,5 \\
\hline Ardea alba (Linnaeus, 1758) & 19 & 5 & 4 & 16 & 44 & 0,46 & 7,4 \\
\hline Syrigma sibilatrix (Temminck, 1824) & 10 & 6 & 3 & 7 & 26 & 0,27 & 5,5 \\
\hline Egretta thula (Molina, 1782) & 9 & 1 & 3 & 19 & 32 & 0.33 & 5,1 \\
\hline \multicolumn{8}{|l|}{ Threskioniithidae } \\
\hline Plegadis chihi (Vieillot, 1817) & 16 & 7 & 0 & 84 & 107 & 1,11 & 6,5 \\
\hline Phimosus infuscatus (Lichtenstein, 1823) & 3 & 37 & 2 & 14 & 56 & 0,58 & 4,2 \\
\hline \multicolumn{8}{|l|}{ Ciconiidae } \\
\hline Ciconia maguari (Gmelin, 1789) & 1 & 0 & 0 & 0 & 1 & 0,01 & 0,9 \\
\hline Mycteria americana (Linnaeus, 1758) & 3 & 0 & 0 & 0 & 3 & 0,03 & 0,5 \\
\hline \multicolumn{8}{|l|}{ Cathartiformes } \\
\hline \multicolumn{8}{|l|}{ Cathartidae } \\
\hline Cathartes aura (Linnaeus, 1758) & 13 & 1 & 4 & 0 & 18 & 0,18 & 5,6 \\
\hline Coragyps atratus (Bechstein, 1793) & 2 & 0 & 0 & 2 & 4 & 0,04 & 0,9 \\
\hline
\end{tabular}

$\mathrm{X}$ : Valores não quantificados 
Tabela 1. Continuação...

\begin{tabular}{|c|c|c|c|c|c|c|c|}
\hline \multirow{2}{*}{ Espécies } & \multicolumn{5}{|c|}{ Estações } & \multirow{2}{*}{$\operatorname{AR}(\%)$} & \multirow{2}{*}{ FO $(\%)$} \\
\hline & Primavera & Verão & Outono & Inverno & Total Anual & & \\
\hline \multicolumn{8}{|l|}{ Falconiformes } \\
\hline \multicolumn{8}{|l|}{ Accipitridae } \\
\hline Rupornis magnirostris (Gmelin, 1788) & 0 & 1 & 3 & 0 & 4 & 0,04 & 0,9 \\
\hline \multicolumn{8}{|l|}{ Falconidae } \\
\hline Caracara plancus (Miller, 1777) & 7 & 7 & 2 & 8 & 24 & 0,25 & 8,3 \\
\hline Milvago chimachima (Vieillot, 1816) & 0 & 0 & 2 & 2 & 4 & 0,04 & 1,8 \\
\hline Milvago chimango (Vieillot, 1816) & 6 & 3 & 3 & 9 & 21 & 0,22 & 6,5 \\
\hline Falco sparverius (Linnaeus, 1758) & 0 & 1 & 1 & 1 & 3 & 0,03 & 1,4 \\
\hline \multicolumn{8}{|l|}{ Gruiformes } \\
\hline \multicolumn{8}{|l|}{ Rallidae } \\
\hline Gallinula galeata (Lichtenstein, 1818) & 0 & 2 & 0 & 3 & 5 & 0,05 & 3,2 \\
\hline \multicolumn{8}{|l|}{ Charadriiformes } \\
\hline \multicolumn{8}{|l|}{ Charadriidae } \\
\hline Vanellus chilensis (Molina, 1782) & 116 & 82 & 104 & 123 & 425 & 4,43 & 31,9 \\
\hline \multicolumn{8}{|l|}{ Recurvirostridae } \\
\hline Himantopus melanurus (Vieillot, 1817) & 0 & 22 & 0 & 0 & 22 & 0,23 & 0,9 \\
\hline \multicolumn{8}{|l|}{ Jacanidae } \\
\hline Jacana jacana (Linnaeus, 1766) & 4 & 7 & 1 & 4 & 16 & 0,17 & 3,7 \\
\hline \multicolumn{8}{|l|}{ Laridae } \\
\hline Chroicocephalus maculipennis (Lichtenstein, 1823) & 0 & 0 & 0 & 94 & 94 & 0,98 & 8,8 \\
\hline Chroicocephalus cirrocephalus (Vieillot, 1818) & 28 & 2 & 6 & 39 & 75 & 0,78 & 3,2 \\
\hline \multicolumn{8}{|l|}{ Sternidae } \\
\hline Phaetusa simplex (Gmelin, 1789) & 0 & 0 & 0 & 28 & 28 & 0,29 & 4,2 \\
\hline Sterna trudeaui (Audubon, 1838) & 0 & 0 & 3 & 2 & 5 & 0,05 & 1,8 \\
\hline \multicolumn{8}{|l|}{ Columbiformes } \\
\hline \multicolumn{8}{|l|}{ Columbidae } \\
\hline Columbina talpacoti (Temminck, 1811) & 20 & 70 & 37 & 39 & 166 & 1,73 & 36,1 \\
\hline Columbina picui (Temminck, 1813) & 57 & 97 & 122 & 69 & 345 & 3,59 & 54,2 \\
\hline Columba livia (Gmelin, 1789) & 353 & 278 & 320 & 225 & 1.176 & 12,26 & 58,8 \\
\hline Patagioenas picazuro (Temminck, 1813) & 0 & 0 & 1 & 2 & 3 & 0,03 & 1,4 \\
\hline Zenaida auriculata (Des Murs, 1847) & 4 & 2 & 8 & 45 & 59 & 0,61 & 16,2 \\
\hline Leptotila verreauxi (Bonaparte, 1855) & 12 & 12 & 10 & 2 & 36 & 0,37 & 8,8 \\
\hline Psittaciformes & & & & & & & \\
\hline Psittacidae & & & & & & & \\
\hline Myiopsitta monachus (Boddaert, 1783) & 320 & 346 & 292 & 260 & 1.218 & 12,70 & 49,1 \\
\hline Cuculiformes & & & & & & & \\
\hline Cuculidae & & & & & & & \\
\hline Guira guira (Gmelin, 1788) & 46 & 54 & 23 & 54 & 177 & 1,84 & 24,5 \\
\hline Tapera naevia (Linnaeus, 1766) & 0 & 0 & 0 & 1 & 1 & 0,01 & 0,5 \\
\hline Strigiformes & & & & & & & \\
\hline Strigidae & & & & & & & \\
\hline Athene cunicularia (Molina, 1782) & 0 & 2 & 0 & 1 & 3 & 0,03 & 0,9 \\
\hline Apodiformes & & & & & & & \\
\hline Trochilidae & & & & & & & \\
\hline Florisuga fusca (Vieillot, 1817) & 0 & 0 & 4 & 2 & 6 & 0,06 & 0,5 \\
\hline Hylocharis chrysura (Shaw, 1812) & 7 & 18 & 15 & 40 & 80 & 0,83 & 29,2 \\
\hline Leucochloris albicollis (Vieillot, 1818) & 1 & 4 & 2 & 0 & 7 & 0,07 & 3,2 \\
\hline Piciformes & & & & & & & \\
\hline Picidae & & & & & & & \\
\hline Veniliornis spilogaster (Wagler, 1827) & 0 & 0 & 2 & 0 & 2 & 0,02 & 0,9 \\
\hline Colaptes melanochloros (Gmelin, 1788) & 4 & 5 & 3 & 2 & 14 & 0,01 & 4,2 \\
\hline Colaptes campestris (Vieillot, 1818) & 13 & 8 & 12 & 16 & 49 & 0,51 & 10,6 \\
\hline Passeriformes & & & & & & & \\
\hline Furnariidae & & & & & & & \\
\hline
\end{tabular}


Tabela 1. Continuação...

\begin{tabular}{|c|c|c|c|c|c|c|c|}
\hline \multirow{2}{*}{ Espécies } & \multicolumn{5}{|c|}{ Estações } & \multirow{2}{*}{$\operatorname{AR}(\%)$} & \multirow{2}{*}{ FO $(\%)$} \\
\hline & Primavera & Verão & Outono & Inverno & Total Anual & & \\
\hline Furnarius rufus (Gmelin, 1788) & 283 & 257 & 289 & 182 & 1.011 & 10,53 & 94,9 \\
\hline \multicolumn{8}{|l|}{ Tyrannidae } \\
\hline Serpophaga subcristata (Vieillot, 1817) & 0 & 0 & 2 & 0 & 2 & 0,02 & 0,9 \\
\hline Phylloscartes ventralis (Temminck, 1824) & 1 & 0 & 0 & 4 & 5 & 0,05 & 0,9 \\
\hline Satrapa icterophrys (Vieillot, 1818) & 1 & 0 & 0 & 0 & 1 & 0,01 & 0,5 \\
\hline Xolmis irupero (Vieillot, 1823) & 5 & 7 & 3 & 4 & 19 & 0,20 & 7,4 \\
\hline Machetornis rixosa (Vieillot, 1819) & 55 & 37 & 165 & 47 & 304 & 3,17 & 51,4 \\
\hline Pitangus sulphuratus (Linnaeus, 1766) & 510 & 441 & 436 & 344 & 1.731 & 18,04 & 99,4 \\
\hline Tyrannus melancholicus (Vieillot, 1819) & 1 & 0 & 0 & 0 & 1 & 0,01 & 0,5 \\
\hline Tyrannus savana (Vieillot, 1808) & 54 & 3 & 0 & 1 & 58 & 0,60 & 19,4 \\
\hline \multicolumn{8}{|l|}{ Vireonidae } \\
\hline Cyclarhis gujanensis (Gmelin, 1789) & 3 & 0 & 2 & 0 & 5 & 0,05 & 2,3 \\
\hline \multicolumn{8}{|l|}{ Hirundinidae } \\
\hline Pygochelidon cyanoleuca (Vieillot, 1817) & 229 & 70 & 46 & 124 & 469 & 4,88 & 59,9 \\
\hline Progne tapera (Vieillot, 1817) & 3 & 10 & 0 & 1 & 14 & 0,15 & 0,5 \\
\hline Tachycineta leucorrhoa (Vieillot, 1817) & 68 & 10 & 1 & 26 & 105 & 1,09 & 20,4 \\
\hline Hirundo rustica (Linnaeus, 1758) & 0 & 4 & 0 & 0 & 4 & 0,04 & 0,9 \\
\hline \multicolumn{8}{|l|}{ Troglodytidae } \\
\hline Troglodytes musculus (Naumann, 1823) & 93 & 65 & 28 & 57 & 243 & 2,53 & 50 \\
\hline \multicolumn{8}{|l|}{ Turdidae } \\
\hline Turdus rufiventris (Vieillot, 1818) & 55 & 15 & 22 & 24 & 116 & 1,21 & 24,5 \\
\hline Turdus amaurochalinus (Cabanis, 1850) & 2 & 2 & 25 & 25 & 54 & 0,56 & 14,4 \\
\hline Turdus albicollis (Vieillot, 1818) & 0 & 0 & 3 & 0 & 3 & 0,03 & 0,5 \\
\hline \multicolumn{8}{|l|}{ Mimidae } \\
\hline Mimus saturninus (Lichtenstein, 1823) & 5 & 0 & 0 & 1 & 6 & 0,06 & 0,9 \\
\hline \multicolumn{8}{|l|}{ Coerebidae } \\
\hline Coereba flaveola (Linnaeus, 1758) & 5 & 2 & 12 & 19 & 38 & 0,40 & 12,5 \\
\hline \multicolumn{8}{|l|}{ Thraupidae } \\
\hline Tangara sayaca (Linnaeus, 1766) & 136 & 15 & 23 & 65 & 239 & 2,49 & 52,8 \\
\hline Pipraeidea bonariensis (Gmelin, 1789) & 1 & 0 & 0 & 4 & 5 & 0,05 & 2,3 \\
\hline Stephanophorus diadematus (Temminck, 1823) & 0 & 0 & 2 & 0 & 2 & 0,02 & 0,5 \\
\hline \multicolumn{8}{|l|}{ Emberezidae } \\
\hline Zonotrichia capensis (Statius Muller, 1776) & 13 & 5 & 5 & 12 & 35 & 0,36 & 5,5 \\
\hline Poospiza nigrorufa (d'Orbigny \& Lafresnaye, 1837) & 0 & 0 & 1 & 0 & 1 & 0,01 & 0,5 \\
\hline Sicalis flaveola (Linnaeus, 1766) & 29 & 9 & 15 & 2 & 55 & 0,57 & 16,7 \\
\hline Embernagra platensis (Gmelin, 1789) & 0 & 0 & 0 & 3 & 3 & 0,03 & 0,5 \\
\hline Volatinia jacarina (Linnaeus, 1766) & 1 & 0 & 0 & 0 & 1 & 0,01 & 0,5 \\
\hline Paroaria coronata (Miller, 1776) & 4 & 1 & 0 & 2 & 7 & 0,07 & 1,8 \\
\hline \multicolumn{8}{|l|}{ Parulidae } \\
\hline Parula pitiayumi (Vieillot, 1817) & 1 & 1 & 2 & 5 & 9 & 0,09 & 4,2 \\
\hline Basileuterus culicivorus (Deppe, 1830) & 2 & 13 & 3 & 0 & 18 & 0,19 & 6,5 \\
\hline Basileuterus leucoblepharus (Vieillot, 1817) & 0 & 4 & 2 & 0 & 6 & 0,06 & 1,4 \\
\hline \multicolumn{8}{|l|}{ Icteridae } \\
\hline Icterus cayanensis (Linnaeus, 1766) & 1 & 0 & 1 & 2 & 4 & 0,04 & 1,4 \\
\hline Amblyramphus holosericeus (Scopoli, 1786) & 0 & 1 & 0 & 0 & 1 & 0,01 & 0,9 \\
\hline Chrysomus ruficapillus (Vieillot, 1819) & 50 & 8 & 5 & 105 & 168 & 1,75 & 19,4 \\
\hline Pseudoleistes guirahuro (Vieillot, 1819) & 2 & 0 & 0 & 7 & 9 & 0,09 & 1,4 \\
\hline Agelaioides badius (Vieillot, 1819) & 1 & 9 & 4 & 1 & 15 & 0,16 & 3,2 \\
\hline Molothrus bonariensis (Gmelin, 1789) & 224 & 49 & 58 & 72 & 403 & 4,2 & 36,1 \\
\hline \multicolumn{8}{|l|}{ Passeridae } \\
\hline Passer domesticus (Linnaeus, 1758) & $\mathrm{X}$ & $\mathrm{X}$ & $\mathrm{X}$ & $\mathrm{X}$ & $\mathrm{X}$ & $\mathrm{X}$ & 95,4 \\
\hline Número Total de Contatos & 2.936 & 2.150 & 2.149 & 2.360 & 9.595 & & \\
\hline Número Total de Espécies & 58 & 56 & 55 & 61 & 84 & & \\
\hline
\end{tabular}

$\mathrm{X}$ : Valores não quantificados 
Quanto à estruturação da assembleia em termos de abundância relativa, 72 espécies $(86,7 \%)$ apresentaram menos de 200 contatos; sete $(8,4 \%)$ apresentaram entre 200 e 1.000 contatos; e quatro espécies $(4,8 \%)$ apresentaram mais de 1.000 contatos. As espécies com mais de 1.000 contatos foram Furnarius rufus (n=1.011; 10,5\%), Columba livia $(\mathrm{n}=1.176 ; 12,2 \%)$, Myiopsitta monachus $(\mathrm{n}=1.218 ; 12,7 \%) \mathrm{e}$ Pitangus sulphuratus $(\mathrm{n}=1.731 ; 18 \%)$ (Tabela 1). Durante o período de um ano, foram contabilizados 5.136 contatos destas quatro espécies, o que representou $53,5 \%$ do total da amostra. Considerandose apenas as 72 espécies da amostra com menos de 200 contatos, 46 espécies $(63,9 \%)$ apresentaram menos de 20 contatos registrados e para 26 espécies $(36,1 \%)$ foram obtidos de 21 a 200 contatos. Foram registrados apenas um indivíduo de Ardea cocoi, Ciconia maguari, Tapera naevia, Satrapa icterophrys, Tyrannus melancholicus, Poospiza nigrorufa, Volatinia jacarina e Amblyramphus holosericeu, durante todo o período de amostragem (Tabela 1).

Em relação à frequência de ocorrência das 84 espécies, 67 (78,8\%) estiveram presentes em menos do que $20 \%$ dos pontos amostrados; sete $(8,3 \%)$ espécies foram registradas entre 20 e $40 \%$ e entre 40 e $60 \%$ dos pontos; e apenas três $(3,6 \%)$ espécies foram visualizadas entre 80 e $100 \%$ dos pontos. Nenhuma espécie obteve FO entre 60 e $80 \%$ dos pontos. As espécies com maior FO foram P. sulphuratus (99,4\%), Passer domesticus (95,4\%) e F. rufus (94,9\%). Treze espécies foram registradas em um único ponto fixo de observação.

A riqueza total de aves variou de 55 a 61 espécies entre as estações. O maior número de espécies foi registrado no inverno $(n=61 ; 72,6 \%)$, seguido da primavera $(n=58 ; 69 \%)$, verão $(n=56$; $66,6 \%)$ e outono $(\mathrm{n}=55 ; 65,5 \%)$. Das 84 espécies registradas, 21 (25\%) estiveram presentes em somente uma estação, 17 (20,2\%) em duas estações, nove $(10,7 \%)$ em três estações e 37 espécies $(44,1 \%)$ estiveram presentes em todas as estações. No verão de 2007/ 2008, foram registradas 11 espécies que não haviam sido registradas na primavera de 2007, por outro lado, 13 espécies presentes na primavera 2007 não foram registradas no verão. No outono de 2008, 11 espécies a mais foram registradas em relação ao verão 2007/2008 e 12 não foram registradas. No inverno de 2008 , foram registradas 18 espécies que não haviam sido contabilizadas no outono de 2008 e 12 espécies não foram visualizadas comparando-se as duas estações.

A estação em que se obteve maior abundância relativa foi a primavera $(n=2.936 ; 30,6 \%)$, seguida do inverno $(n=2360 ; 24,6 \%)$, verão $(\mathrm{n}=2150 ; 22,4 \%)$ e outono $(\mathrm{n}=2149 ; 22,4 \%)$. As quatro espécies de aves mais abundantes na amostra, F. rufus, C. livia, M. monachus e P. sulphuratus, apresentaram abundâncias relativas altas e variáveis entre as quatro estações do ano. O número de indivíduos contabilizados de P. sulphuratus foi máximo na primavera de 2007 e declinou até o inverno 2008 e para as outras três espécies a variação do número de indivíduos parece ter sido aleatória (Tabela 1).

\section{Discussão}

Os dados de riqueza obtidos em estudos que analisaram a assembleia de aves em áreas urbanas de cidades do RS e do Brasil são bastante variados entre si e em relação ao resultado do presente estudo. No RS, foram registradas 24 espécies nos Balneários de Tramandaí e Imbé (Ramos \& Daudt 2005); 170 espécies foram registradas em diferentes parques de Porto Alegre (Scherer et al. 2005), enquanto 132 espécies, de 43 famílias, foram registradas considerando toda a malha urbana da cidade (Fontana et al. 2011); 170 espécies foram contabilizadas em uma área verde em Caxias do Sul (Silva 2006) e 34 espécies de aves foram amostradas em Uruguaiana (Paetzold \& Querol 2008). No Brasil, os estudos realizados em cidades foram concentrados em áreas verdes, como parques, remanescentes florestais e entorno de lagos. Como exemplos podem ser citados os estudos realizados em Maringá (PR), com 144 espécies de aves registradas (Krügel \& Anjos 2000); Vitória (ES), com 120 espécies contabilizadas (Simon et al. 2007); Uberlândia (MG), no Parque Municipal do Sabiá, onde foram registradas 149 espécies (Franchin \& Marçal Júnior 2004), sendo que na mesma cidade foram registradas 66 espécies de aves quando o levantamento foi realizado em avenidas (Torga et al. 2007); Ipatinga (MG), com 57 espécies amostradas (Fuscaldi \& Loures-Ribeiro 2008) e Iporá (GO) com 70 espécies contabilizadas (Silva \& Blamires 2007). Riquezas de aves menores do que a obtida em Pelotas foram registradas em trabalhos realizados em cidades situadas em latitudes maiores no Uruguai e na Argentina. Na cidade de Mar del Plata, foram constatadas 31 espécies (Leveau \& Leveau 2004); em Buenos Aires, foram registradas 49 espécies (Faggi \& Perepelizin 2006); e na cidade de Mendoza, foram contabilizadas 63 espécies (Gómez 2006).

A comparação de dados de riqueza total obtidos em estudos realizados em diferentes pontos geográficos apresenta uma série de dificuldades relacionadas ao fato da riqueza ser uma característica de assembleias condicionada por múltiplos fatores. A riqueza de aves em ambiente urbano pode ser influenciada, em primeiro lugar, pela latitude, ou seja, a posição geográfica da cidade, e por características regionais como a altitude, geomorfologia e vegetação característica. Clergeau et al. (2006) estudaram os efeitos da urbanização na avifauna em 19 cidades da Itália, França e Finlândia selecionadas ao longo de um gradiente de latitude. O número de espécies foi analisado em um gradiente de urbanização para todas as cidades, incluindo três diferentes setores. Os autores concluíram que a latitude explica $89 \%$ das diferenças na riqueza encontradas no setor periurbano, mas somente $52 \%$ no setor central. Estes dados demonstram que apesar da latitude influenciar a riqueza de espécies em áreas urbanas, seu efeito é atenuado pela urbanização. Em segundo lugar, podem exercer influencia o tamanho da cidade, sua heterogeneidade em termos de habitas e micro-habitats e a qualidade e heterogeneidade dos habitats de entorno. Por exemplo, Leveau \& Leveau (2006) trabalharam em três cidades costeiras de diferentes tamanhos na Argentina, obtendo a maior riqueza total de espécies $(n=14)$ na menor cidade (Miramar), onde também foi maior a riqueza e abundância de espécies não associadas ao homem. A diferença no esforço amostral também pode ser um fator determinante para explicar a variação nas riquezas observadas em áreas urbanas, assim como as diferenças na metodologia aplicada e a questão enfocada no trabalho.

Desta forma, quando o objetivo é discutir a riqueza de espécies da matriz urbana de uma cidade é mais apropriado a comparação com dados de estudos realizados no seu entorno ou na região, que podem esclarecer quantas espécies foram perdidas com a urbanização e, principalmente, quantas espécies presentes na região suportam diferentes graus de urbanização. As 84 espécies de aves contabilizadas neste estudo representam $12,7 \%$ das aves listadas para o RS por Bencke et al. (2010) e 21,8\% das 385 espécies de aves presentes na porção brasileira do Bioma Pampa (Bilenca \& Miñarro 2004). Através destas comparações percebe-se que a riqueza de aves na área urbana de Pelotas é alta, principalmente quando consideramos que se trata de uma pequena área geográfica, com grau de urbanização e ocupação humana relativamente altos. Além disso, deve-se considerar que a área onde a cidade de Pelotas está inserida tem um longo histórico de colonização, destruição e alteração de habitat, ou seja, já deve ter ocorrido um processo histórico de perda de espécies.

Quando se trata da distribuição de aves em áreas urbanas tanto a escala espacial local quanto a de paisagem são importantes (Melles et al. 2003), apesar de alguns estudos apresentarem evidências de que a paisagem de entorno (periurbana) seja menos importante do que características locais na determinação da riqueza de aves (Clergeau et al. 2001). A riqueza de espécies relativamente alta 
na matriz urbana de Pelotas pode ser influenciada positivamente pelo entorno bastante diversificado da cidade, o qual é composto por um mosaico de diferentes paisagens, incluindo áreas utilizadas para a agropecuária, principalmente plantação de arroz, criação de gado e silvicultura, habitats lacustres extensos e relativamente bem preservados e resquícios de campos, banhados e florestas nativas. Esses diferentes habitats podem manter populações estabelecidas de uma gama muito diversificada de espécies, cujos indivíduos podem circular pela matriz urbana, principalmente em áreas periféricas. Outra possibilidade é que certas espécies de entorno tenham também populações estabelecidas em áreas suburbanas e periurbanas, onde encontram abrigo, alimento e sítios para nidificação.

Por outro lado, a riqueza e a diversidade de aves na cidade de Pelotas podem estar sendo influenciadas negativamente por atributos locais do habitat. Pelotas possui um número muito reduzido de praças, não possui parques, reservas de vegetação nativa e lagos e, aparentemente, a arborização urbana não é significativa, principalmente nas zonas centrais e suburbanas, ponto que deve ser investigado qualitativamente e quantitativamente. As aves respondem a composição e estrutura da vegetação e áreas urbanas que mantêm as características da vegetação nativa retém mais espécies nativas de aves que as que não mantêm (Chace \& Walsh 2006). Em estudo realizado em Mar Del Plata, na Argentina, Leveau \& Leveau (2004) constataram que a riqueza, a abundância e a diversidade de aves são maiores em áreas suburbanas e são significativamente correlacionadas com a proporção de árvores, arbustos e gramados. Muitas espécies vegetais utilizadas na área urbana de Pelotas são exóticas, o que também pode exercer efeitos sobre a assembleia de aves. White et al. (2005) avaliaram quatro diferentes tipos de habitats na área urbana de Melbourne, na Autrália, incluindo parques com vegetação nativa remanescente, ruas com vegetação nativa, ruas com vegetação exótica e ruas em áreas residenciais recentes sem vegetação madura. A riqueza e a abundância foram menores nas ruas arborizadas com espécies exóticas e recém urbanizadas do que em parques e ruas com espécies nativas. Além disso, há menos aves introduzidas em parques e ruas com vegetação nativa do que em ruas arborizadas com espécies vegetais exóticas e ruas de bairros recentes.

Durante os 12 meses de monitoramento foram contabilizados quase 10.000 registros de aves, o que pode ser considerado um número bastante alto para área urbana. Esse resultado condiz com o padrão já observado em outros estudos que descrevem que a biomassa de aves é maior em áreas urbanizadas (Chace \& Walsh 2006). A assembleia de aves de Pelotas caracteriza-se também pela dominância em termos numéricos de poucas espécies que apresentaram FO extremamente altas, principalmente F. rufus, C. livia, M. monachus e P. sulphuratus. Vários outros estudos já haviam constatado sua presença em altas abundâncias em cidades na região Neotropical (Jebai et al. 2009, Leveau \& Leveau 2004, Ruszczyk et al. 1987). Columba livia é uma indicadora de qualidade ambiental negativa, pois foi encontrada em maior abundância em áreas mais intensamente urbanizadas, com maior movimentação de pessoas e veículos na área urbana de Uberlândia, Minas Gerais, sudeste do Brasil (Amâncio et al. 2008). Pitangus sulphuratus apresenta ampla distribuição em todos os níveis de urbanização da cidade de Porto Alegre (RS) e sua abundância é maior na área central e urbanizada da cidade, onde representa mais de $80 \%$ das aves registradas. Em áreas periféricas da cidade, com baixo grau de urbanização, sua abundância é menor que $40 \%$ das aves contabilizadas (Ruszczyk et al. 1987). Porém, em Uberlândia, apesar de ser considerada uma espécie abundante, sua frequência não possui correlação com indicadores de urbanização (fluxo de pessoas e veículos) (Amâncio et al. 2008). Já F. rufus apresenta sua distribuição em Porto Alegre relacionada com áreas com graus menores de urbanização, situadas em torno do núcleo intensamente urbanizado, onde sua frequência é menor do que $10 \%$ dos registros obtidos (Ruszczyk et al. 1987). A existência de várias espécies vegetais frutíferas, muitas destas exóticas, presentes nas calçadas, jardins e pomares, atraem espécies frugívoras como M. monachus (observação pessoal das autoras), que foi a segunda espécie mais abundante em Pelotas.

Estas quatro espécies, juntamente com $P$. domesticus, cuja abundância e FO são altas (A.G. Sacco, F.B. Bergmann \& A.M. Rui, dados não publicados), dominam a assembleia de aves de Pelotas em termos quantitativos. O número de indivíduos de pardais presentes em área urbana em Pelotas é tão elevado que não foi possível realizar uma contagem exata, apenas uma estimativa de número de indivíduos dentro de intervalos de abundância. Fontana et al. (2011) relatou padrão semelhante para Porto Alegre (RS), onde pardais (Passer domesticus) e pombas (Columba livia) são as espécies com o maior número de indivíduos registrados, sendo que espécies introduzidas representam $40 \%$ do total dos indivíduos na amostra.

Por outro lado, 72 espécies tiveram menos do que 200 contatos registrados e 67 espécies ocorreram em menos do que $20 \%$ do total de pontos amostrados, podendo ser consideradas raras na matriz urbana de Pelotas. Os dados indicam que estas espécies possuem distribuição heterogênea, estando, provavelmente, restritas em áreas com grau de urbanização menos intenso ou a micro-habitats raros em áreas urbanas, que oferecem recursos como alimento, abrigo e locais para nidificação. Outro conjunto de espécies raras na amostra é composto por espécies abundantes no entorno de Pelotas que circulam ocasionalmente pelo ambiente urbano ou utilizam as bordas menos urbanizadas deste como, por exemplo, A. cocoi, C. maguari, T. naevia e $P$. nigrorufa (observação pessoal das autoras), as quais tiveram apenas um indivíduo visualizado.

A questão dos fatores que determinam a persistência ou não e abundância de espécies de aves em habitats urbanos tem sido abordada em alguns estudos (Bonier et al. 2007, Croci et al. 2008). Bonier et al. (2007) testaram a hipótese de que aves urbanas tem tolerância ambientais amplas (definida como a habilidade de sobreviver e reproduzir no habitat) em relação a seus congêneres rurais utilizando a distribuição altitude e latitudinal durante o período reprodutivo para a análise. Os autores concluíram que aves urbanas possuem tolerância ambiental ampla em relação a espécies filogeneticamente próximas que ocorrem em apenas em área rurais. Segundo os autores, a flexibilidade no comportamento, fisiologia e ecologia podem contribuir para a habilidade de aves urbanas tolerarem uma ampla gama de condições ambientais, incluindo habitats alterados. As quatro espécies mais abundantes e amplamente distribuídas na matriz urbana de Pelotas se encaixam nessas características, já que possuem ampla distribuição geográfica (Belton 2004), ocorrendo em regiões com condições ambientais muito distintas, com disponibilidade de recursos muito variados.

Quanto à sazonalidade, a riqueza total das aves foi pouco variável entre as estações do ano. A maior riqueza de espécies no inverno $(n=61)$ pode ser explicada pela presença de espécies aquáticas, como Phalacrocorax brasilianus, Chroicocephalus cirrocephalus e Phaetusa simplex, que podem estar presentes na cidade devido à disponibilidade de abrigo ou alimento. A alta riqueza na primavera $(n=58)$ era um resultado esperado, já que nesta estação existe maior disponibilidade de recursos e é a época em que as aves estão mais ativas devido à temporada de reprodução, tendo maior probabilidade de serem registradas. Também estiveram presentes algumas espécies migrantes, como Hirundo rustica, Mycteria americana e Tyrannus melancholicus (Belton 2004). Na primavera, também houve o maior número de contatos registrados (2.936), o que corresponde a $30 \%$ do total, incremento relacionado a um aumento real do número 
de indivíduos nas populações, com o nascimento e recrutamento de filhotes.

A composição da assembleia de aves no que se refere à presença $\mathrm{e}$ ausência de espécies entre as estações foi variável. Este resultado pode ser atribuído ao fato de que algumas espécies permanecem na área urbana em apenas parte do ano, mas também a probabilidade baixa de indivíduos de espécies raras e de indivíduos de espécies residentes apenas no entorno da cidade serem visualizados. Porém, apenas um estudo incluindo vários anos de monitoramento poderia esclarecer o real padrão de composição da assembleia. Das 37 espécies presentes em todas as estações do ano, para 16 espécies foram contabilizados mais do que 100 indivíduos e apenas para 11 espécies foram contabilizados mais do que 200 indivíduos. A identificação destas espécies residentes, abundantes e com frequências de ocorrência altas na matriz urbana é de extrema importância, já que elas podem possuir potencial como indicadoras da qualidade do habitat urbano e de diferentes níveis de urbanização (Amâncio et al. 2008). O estabelecimento de programas de monitoramentos populacionais de longo prazo de espécies de aves torna possível à detecção de alterações na qualidade da matriz urbana em diferentes escalas espaciais e temporais.

A assembleia de aves da cidade de Pelotas apresentou riqueza representativa se comparada com a região, porém poucas espécies são realmente abundantes e bem distribuídas. Esse padrão pode ser reflexo da baixa qualidade e heterogeneidade da matriz urbana, que possui poucas praças, parques, não possui áreas de reserva de vegetação nativa, nem lagos e possui baixo grau de arborização nas ruas e avenidas. Porém, deve ser realizada uma análise qualitativa e quantitativa das características da matriz urbana para verificar sua influência sobre a assembleia de aves. Ações para promover o incremento da diversidade e abundância de aves em ecossistemas urbanos devem ser aplicadas em diferentes escalas espaciais, como as paisagens do entorno da cidade, na própria cidade, em setores da cidade e em propriedades particulares. Entre as recomendações mais específicas para a promoção da diversidade urbana estão incluídos o plantio de árvores e arbustos, a disponibilização de ninhos artificiais e comedouros para pássaros, a regulação do comportamento humano e a criação, restauração e manejo de áreas naturais (Savard et al. 2000). No caso de Pelotas, programas de arborização urbana com espécies nativas, criação de áreas verdes, preservação de áreas úmidas na área urbana e no seu entorno, provavelmente, seriam efetivas.

\section{Referências Bibliográficas}

ALVES, M.A.S. \& PEREIRA, E.F. 1998. Richness, abundance and seasonality of bird species in a lagoon of an urban area (Lagoa Rodrigo de Freitas) of Rio de Janeiro, Brazil. Ararajuba 6:110-116.

AMÂNCIO, S., SOUZA, V.B. \& MELO, C. 2008. Columbia livia e Pitangus sulphuratus como indicadoras de qualidade ambiental em área urbana. Rev. Bras. Ornitol. 16(1):32-37.

BELTON, W. 2004. Aves silvestres do Rio Grande do Sul. 4. ed. atual Fundação Zoobotânica do Rio Grande do Sul, Porto Alegre, 175p.

BENCKE, G.A., DIAS, R.A., BUGONI, L., AGNES, C.E., FONTANA, C.S., MAURÍCIO, G.N. \& MACHADO, D.B. 2010. Revisão e atualização da lista das aves do Rio Grande do Sul, Brasil. Iheringia, Zool. 100(4):391-402. http://dx.doi.org/10.1590/S007347212010000400014

BIBBY, C.J., BURGESS, N.D. \& HILL, D.A. 1992. Bird Censos Techniques. Royal Society for the Protection of Birds, British trust for Ornithology, Academic Press and The University Press, Cambridge, 257p.

BILENCA, D.N. \& MIÑARRO, F.O. 2004. Identificación de Áreas Valiosas de Pastizal (AVPs) em las Pampas y Campos de Argentina, Uruguay y sur de Brasil. Fundación Vida Silvestre, Buenos Aires, 323p.
BLAIR, R.B. 2001. Creating a homogeneous avifauna. In Avian ecology and conservation in an urbanizing world (J.M. Marsluff, R. Bowman \& R. Donnelly, ed.). Kluwer Academic Publishers, Boston, p.459-486. http:// dx.doi.org/10.1007/978-1-4615-1531-9_22

BONIER, F., MARTIN, P.R. \& WINGFIELD, J.C. 2007. Urban birds have broader environmental tolerance. Biol. Lett. 3:670-673. http://dx.doi. org/10.1098/rsbl.2007.0349

BORGES, S.H. \& GUILHERME, E. 2000. Comunidade de aves em um fragmento florestal urbano em Manaus, Amazonas, Brasil. Ararajuba 8:17-23.

CHACE, J.F. \& WALSH, J.J. 2006. Urban effects on native avifauna: a review. Landscape Urban Plan. 74: 46-69. http://dx.doi.org/10.1016/j. landurbplan.2004.08.007

CLERGEAU, P., SAVARD, J.-P.L., MENNECHEZ, G., FALARDEAU, G. 1998. Bird abundance and diversity along an urban-rural gradient: a comparative study between two cities on different continents. Condor 100:413-425. http://dx.doi.org/10.2307/1369707

CLERGEAU, P., JOKIMÄKI, J. \& SAVARD, J.-P.L. 2001. Are urban bird communities influenced by the bird diversity of adjacent landscapes? J. Appl. Ecol. 38:1122-1134. http://dx.doi.org/10.1046/j.13652664.2001.00666.x

CLERGEAU, P., CROCI, S., JOKIMÄKI, J., KAISANLAHTI-JOKIMÄKI, M. \& DINETTI, M. 2006. Avifauna homogenisation by urbanisation: analysis at different European latitudes. Biol. Conserv. 127:336-344. http://dx.doi.org/10.1016/j.biocon.2005.06.035

Comitê Brasileiro de Registros Ornitológicos. 2011. Listas das aves do Brasil. 10. ed. http://www.cbro.org.br>. (último acesso em 29/09/2011).

CROCI, S., BUTET, A. \& CLERGEAU, P. 2008. Does urbanization filter birds on the basis of their biological traits? The Condor 110(2):223-240. http://dx.doi.org/10.1525/cond.2008.8409

EMBRAPA. Normais climatológicas (1971/2000) realizadas pela Embrapa (Estação Agroclimatológica de Pelotas - Capão do Leão) com convênio UFPel e INMET. http://www.cpact.embrapa.br/agromet/estacao/normais. html. (último acesso em 15/05/2008).

FAGGI, A. \& PEREPELIZIN, V. 2006. Riqueza de aves a lo largo de um gradiente de urbanización em la ciudad de Buenos Aires. Ver. Mus. Argentino. Cienc. Nat. 8(2):289-297.

FONTANA, C.S., BURGER, M.I. \& MAGNUSSON, W.E. 2011. Bird diversity in a subtropical South-American City: effects of noise levels, arborisation and human population density. Urban Ecosyst 14:341-360. http://dx.doi.org/10.1007/s11252-011-0156-9

FRANCHIN, A.G. \& MARÇAL JÚNIOR, O. 2004. A riqueza da avifauna no Parque Municipal do Sabiá, zona urbana de Uberlândia (MG) Biotemas 17(1):179-202.

FUSCALDI, R.G. \& LOURES-RIBEIRO, A. 2008. A avifauna de uma área urbana do município de Ipatinga, Minas Gerais, Brasil. Biotemas 21(3):125-133. http://dx.doi.org/10.5007/21757925.2008v21n3p125

GÓMEZ, V.E. 2006. Aves del Parque General San Martín (Mendoza). Distribuicíon y Características. Multequina 15:81-95.

HAMMER, O., HARPER, D.A.T. \& RYAN, P.D. 2001. Paleontological Statistics - PAST. Version 2.09. http://folk.uio.no/ohammer/past.

INSTITUTO BRASILEIRO DE GEOGRAFIA E ESTATÍSTICA- IBGE 1986. Levantamento de recursos naturais (Folha SH.22 Porto Alegre e parte das Folhas SH.21 Uruguaiana e SI.22 Lagoa Mirim). IBGE, Rio de Janeiro, CD-ROM.

INSTITUTO BRASILEIRO DE GEOGRAFIAE ESTATÍSTICA-IBGE. 2007. Contagem da população 2007 - Pelotas/RS. http://www.ibge.gov.br/home/ estatistica/populacao/contagem2007 (último acesso em 19/12/2007).

JEBAI, G.T., ARAKAKI, B.R., SILVA, C.A.P., SOUZA, A.R., GOMES, T.M. \& ANJOS, L. 2009. Análise comparativa da densidade de onze passeriformes em duas áreas urbanas em Londrina, norte do Paraná, Brasil. Rev. Bras. Ornitol. 17(3-4):183-186. 
KRÜGEL, M.M. \& ANJOS, L. 2000. Bird communities in forest remnants in the city of Maringa, Paraná State, Southern Brazil. Ornitol. Neotrop. 11:315-330.

LEVEAU, L.M. \& LEVEAU, C.M. 2004. Comunidades de aves en un gradiente urbano de la ciudad de Mar Del Plata, Argentina. Hornero 19(1):13-21.

LEVEAU, C.M. \& LEVEAU, L.M. 2006. Ensambles de aves en calles arboladas de tres ciudades costeras del sudeste de la provincial de Buenos Aires, Argentina. Hornero 21(1):25-30.

MATARAZZO-NEUBERGER, W.M. 1992. Avifauna urbana de dois municípios da grande São Paulo, SP (Brasil). Acta Biol. 21:89-106.

MATARAZZO-NEUBERGER, W.M. 1995. Comunidade de aves de cinco parques e praças da Grande São Paulo, Estado de São Paulo. Ararajuba 3:13-19.

McKINNEY, M.L. 2006. Urbanization as a major cause of biotic homogenization. Biol. Conserv. 127:247-260. http://dx.doi.org/10.1016/j. biocon.2005.09.005

McKINNEY, M.L. 2008. Effects of urbanization on species richness: a review of plants and animals. Urban Ecosyst 11:161-176. http://dx.doi. org/10.1007/s11252-007-0045-4

MELLES, S., GLENN, S. \& MARTIN, K. 2003. Urban bird diversity and landscape complexity: Species-environment associations along a multiscale habitat gradient. Conserv. Ecol. 7(1):5.

MENDONÇA-LIMA, A. \& FONTANA, C.S. 2000. Composição, freqüência e aspectos biológicos da avifauna no Porto Alegre Country Clube, Rio Grande do Sul. Ararajuba 8(1):1-8.

MONTEIRO, M.P. \& BRANDÃO, D. 1995. Estrutura da comunidade de aves do "Campus Samambaia" da Universidade Federal de Goiás, Goiânia, Brasil. Ararajuba 3:21-26.

PAETZOLD, V. \& QUEROL, E. 2008. Avifauna urbana do município de Uruguaiana, RS, Brasil (Resultados Parciais). Biodiversidade Pampeana 6(1):40-45.

RAMOS, L.A. \& DAUDT, R.B. 2005. Avifauna urbana dos balneários de Tramandaí e Imbé, litoral norte do Rio Grande do Sul. Biotemas 18(1):181-191.
REIS, E., LÓPEZ-IBORRA, G.M. \& PINHEIRO, R.T. 2012. Changes in bird species richness through different levels of urbanization: Implications for biodiversity conservation and garden design in Central Brazil. Landscape Urban Plan. 107:31-42. http://dx.doi.org/10.1016/j. landurbplan.2012.04.009

RUSZCZYK, A., RODRIGUES, J.J.S., ROBERTS, T.M.T., BENDATI, M.M.A., DEL PINO, R.S., MARQUES, J.C.V. \& MELO, M.T.Q. 1987. Distribution patterns of eight bird species in the urbanization gradient of Porto Alegre, Brazil. Cienc. Cult. 39(1):14-19.

SAVARD, J.P.L. \& FALLS, B.J. 1982. Influence of habitat structure on the nesting height of birds in urban areas. Can. J. Zool. 59:924-932. http:// dx.doi.org/10.1139/z81-132

SAVARD, J.P.L., CLERGEAU, P. \& MENNECHEZ, G. 2000. Biodiversity concepts and urban ecosystems. Landscape Urban Plan. 48:131-142. http://dx.doi.org/10.1016/S0169-2046(00)00037-2

SCHERER, A., SCHERER, S.B., BUGONI, L., MOHR, L.V., EFE, M.A. \& HARTZ, S.M. 2005. Estrutura trófica da avifauna em oito parques da cidade de Porto Alegre, Rio Grande do Sul, Brasil. Ornithologia 1(1):25-32.

SILVA, F.D.S. \& BLAMIRES, D. 2007. Avifauna urbana no Lago Pôr do Sol, Iporá, Goiás, Brasil. Lundiana 8(1):17-26.

SILVA, R.R.V. 2006. Estrutura de uma comunidade de aves em Caxias do Sul, Rio Grande do Sul, Brasil. Biociências 14(1):27-36.

SILVA, R.R.V. 2007. Assembléia de aves registrada no Lago do Rizzo e seu entorno, em Caxias do Sul, Rio Grande do Sul, Brasil. Atualidades Ornitológicas On-line (137): www.ao.com.br.

SIMON, J.E., LIMA, S.R. \& CARDINALI, T. 2007. Comunidade de aves no Parque Estadual da Fonte Grande, Vitória, Espírito Santo, Brasil. Rev. Bras. Zool. 24(1):121-132. http://dx.doi.org/10.1590/S010181752007000100015

TORGA, K., FRANCHIN, A.G. \& MARÇAL JÚNIOR, O. 2007. A avifauna em uma seção da área urbana de Uberlândia, MG. Biotemas 20(1):7-17.

WHITE, J.G., ANTOS, M.J., FITZSIMONS, J.A. \& PALMER, G.C. 2005. Non-uniform bird assemblages in urban environments: the influence of streetscape vegetation. Landscape Urban Plan. 71(2-4):123-135. http:// dx.doi.org/10.1016/j.landurbplan.2004.02.006 